\title{
Solution of Boundary Value Obstacle Problems Using MQ-RBF and IMQ-RBF
}

\author{
Feng Gao and Chunmei Chi \\ Science School, Qingdao Technological University, Qingdao, Shandong 266033, China \\ Correspondence should be addressed to Feng Gao; gaofeng99@sina.com
}

Received 24 November 2013; Accepted 16 December 2013

Academic Editor: Manyu Xiao

Copyright ( 2013 F. Gao and C. Chi. This is an open access article distributed under the Creative Commons Attribution License, which permits unrestricted use, distribution, and reproduction in any medium, provided the original work is properly cited.

\begin{abstract}
A kind of numerical method which is based on multiquadric RBF, inverse multiquadric RBF, and Wu-Schaback operators is presented for solving second-order and third-order boundary value problems associated with obstacle, unilateral, and contact problems. The algorithms are proved to be highly accurate and easy to implement. Some numerical tests are also presented to show the efficiency of the algorithms.
\end{abstract}

\section{Introduction}

We consider the numerical solutions of the following secondorder boundary value problems:

$$
u^{\prime \prime}(x)= \begin{cases}f(x), & a \leq x \leq c, \\ g(x) u(x)+f(x)+r, & c \leq x \leq d, \\ f(x), & d \leq x \leq b\end{cases}
$$

with the boundary conditions

$$
u(a)=\alpha, \quad u(b)=\beta
$$

and the continuity conditions of $u$ and $u^{\prime}$ at $c$ and $d$. We also consider the following third-order boundary value problems:

$$
u^{\prime \prime \prime}(x)= \begin{cases}f(x), & a \leq x \leq c, \\ g(x) u(x)+f(x)+r, & c \leq x \leq d, \\ f(x), & d \leq x \leq b\end{cases}
$$

with the boundary conditions

$$
u(a)=\alpha_{1}, \quad u^{\prime}(a)=\alpha_{2}, \quad u^{\prime}(b)=\beta
$$

and the continuity conditions of $u, u^{\prime}$, and $u^{\prime \prime}$ at $c$ and $d$. In the two problems, $f(x)$ and $g(x)$ are continuous functions and $\alpha$, $\beta, \alpha_{1}, \alpha_{2}$ are real constants. The two problems arise usually in obstacle, contact, unilateral, and equilibrium problems associated with economics, transportation, oceanography, fluid flow through porous media, and many other fields of pure and applied sciences; see [1-12]. In the literature, some techniques were used to solve the previous two systems of second-order and third-order boundary value problems associated with obstacles. For example, for problem (1), Noor and Khalifa [13] used first-order accuracy collocation method with cubic splines as basis functions. Al-Said et al. [14] developed second- and fourth-order finite difference and spline methods. Al-Said [5] developed and analyzed quadratic and cubic splines methods. He proved that both quadratic and cubic spline methods can be used to produce second-order smooth approximations for the solution of (1) and its first derivative over the whole interval $[a, b]$. Al-Said [7] also used cubic spline polynomial functions for solving problem (1).

For the problem (3), Khalifa and Noor [15] applied quintic spline method for contact problems. Noor and Khalifa [16] applied quartic spline method for odd-order obstacle problems. Gao and Chi [17] applied quartic B-spline method for third-order obstacle problems and Siraj-ul-Islam et al. [12] proposed nonpolynomial spline methods. Some of these methods require huge computational work for finding the numerical solutions.

In this paper, we use multiquadric RBF (MQs) and inverse multiquadric RBF (IMQs) to solve systems of second-order 
and third-order boundary value problems associated with obstacle. Recently, radial base functions (RBF) have been widely applied to the numerical solution of many types of PDE; see $[18,19]$. The advantage of techniques of using RBF is that mesh grid can be avoided and high accuracy can be achieved. However, to the authors' knowledge, little work has been done involving the usage of RBF in the solution of boundary value obstacle problems. Among Radial Basis Functions, MQs and IMQs are especially suitable for solving second-and third-order boundary value problems associated with obstacles because we can integrate IMQs to get MQs. Our technique is that we first use IMQs or MQs quasiinterpolation to approximate the derivatives of the solution of problems of (1) and (3); then we get their integrals to approximate the solutions. The following part of this paper is organized as follows. In Section 2, we review some basics of RBFs. In Section 3, we present the numerical schemes for problem (1) and (3). In Section 4, we do some numerical experiments. Section 5 is our conclusion.

\section{RBF and MQ Quasi-Interpolation}

RBF was proposed in 1951 [20] to deal with geological problem.

For a given region $\Omega \in R^{m}$ and a set distinct interpolation points,

$$
X=\left\{x_{1}, x_{2}, \ldots x_{N}\right\} \subset \Omega .
$$

Given a function $f: X \rightarrow R$, construct the interpolation to $f$

$$
S_{f, X}(x)=\sum_{j=1}^{N} \alpha_{j} \varphi\left(\left\|x-x_{j}\right\|\right), \quad \text { for } x \in \Omega,
$$

where $\|\cdot\|$ denote Euclidean norm and $\varphi$ is a certain RBF. The undetermined coefficients $\left\{\alpha_{1}, \alpha_{2}, \ldots, \alpha_{N}\right\}$ can be obtained by the linear system

$$
\sum_{j=1}^{N} \alpha_{j} \varphi\left(\left\|x_{i}-x_{j}\right\|\right)=f\left(x_{i}\right), \quad i=1,2, \ldots, N .
$$

We adopt multiquadric (MQs) RBF

$$
\phi(r)=\sqrt{s^{2}+r^{2}}
$$

and inverse multiquadric (IMQs) RBF

$$
\varphi(r)=\frac{s^{2}}{\left(s^{2}+r^{2}\right)^{3 / 2}}
$$

to solve second-order and third-order boundary value obstacle problems, where $s \in R^{+}$is a shape parameter. From [21], we know that the linear system (7) has unique solution. It is easy to see that

$$
\left(\sqrt{s^{2}+r^{2}}\right)^{\prime \prime}=\frac{s^{2}}{\left(s^{2}+r^{2}\right)^{3 / 2}} .
$$

We also adopt a kind of Quasi-interpolation to numerically solve problem (1) and (3). The Quasi-interpolation of a univariate function $f:[a, b] \rightarrow R$ with MQs is constructed as follows. On the scattered points

$$
\begin{gathered}
a=x_{0}<x_{1}<\cdots<x_{n}=b, \\
h:=\max _{1 \leq i \leq n}\left(x_{i}-x_{i-1}\right) .
\end{gathered}
$$

Quasi-interpolant of $f$ usually is as follows:

$$
(M f)(x)=\sum_{i=0}^{n} f\left(x_{i}\right) \psi_{i}(x)
$$

where $\psi_{i}(x)$ is linear combinations of the MQs. Consider

$$
\phi_{i}\left(\left|x-x_{i}\right|\right)=\sqrt{s^{2}+\left(x-x_{i}\right)^{2}}, \quad s \in R^{+}
$$

where $s$ is a shape parameter.

Given data $\left\{\left(x_{i}, f_{i}\right)\right\}_{i=1}^{n}$, Wu-Schabacks $L_{D}$ operator is

$$
\left(L_{D} f\right)(x)=\sum_{j=0}^{n} f\left(x_{j}\right) \psi_{j}(x)
$$

where

$$
\begin{gathered}
\psi_{0}(x)=\frac{1}{2}+\frac{\phi_{1}\left(\left|x-x_{1}\right|\right)-\left(x-x_{0}\right)}{2\left(x_{1}-x_{0}\right)}, \\
\psi_{1}(x)=\frac{\phi_{2}\left(\left|x-x_{2}\right|\right)-\phi_{1}\left(\left|x-x_{1}\right|\right)}{2\left(x_{2}-x_{1}\right)} \\
-\frac{\phi_{1}\left(\left|x-x_{1}\right|\right)-\left(x-x_{0}\right)}{2\left(x_{1}-x_{0}\right)}, \\
\psi_{n-1}(x)=\frac{\left(x_{n}-x\right)-\phi_{n-1}\left(\left|x-x_{n-1}\right|\right)}{2\left(x_{n}-x_{n-1}\right)} \\
-\frac{\phi_{n-1}\left(\left|x-x_{n-1}\right|\right)-\phi_{n-2}\left(\left|x-x_{n-2}\right|\right)}{2\left(x_{n-1}-x_{n-2}\right)}, \\
\psi_{n}(x)=\frac{1}{2}+\frac{\phi_{n-1}\left(\left|x-x_{n-1}\right|\right)-\left(x_{n}-x\right)}{2\left(x_{n}-x_{n-1}\right)}, \\
\psi_{j}(x)=\frac{\phi_{j-1}\left(\left|x-x_{j-1}\right|\right)-\phi_{j}\left(\left|x-x_{j}\right|\right)}{2\left(x_{j-1}-x_{j}\right)} \\
-\frac{\phi_{j}\left(\left|x-x_{j}\right|\right)-\phi_{j-1}\left(\left|x-x_{j-1}\right|\right)}{2\left(x_{j}-x_{j-1}\right)},
\end{gathered}
$$

The advantage of $L_{D}$ operator is that high accuracy can be achieved without solving any linear system. 
For $\int \psi_{i}(x) d x$, we need only to integrate $\phi_{i}\left(\left|x-x_{i}\right|\right)$,

$$
\begin{aligned}
\int \phi_{i}\left(\left|x-x_{i}\right|\right) d x \\
=\int \sqrt{s^{2}+\left(x-x_{j}\right)^{2}} d x \\
=\frac{\left(x-x_{j}\right) \sqrt{\left(x-x_{j}\right)^{2}+s^{2}}}{2} \\
\quad+\frac{s^{2}}{2} \ln \left(\left(x-x_{j}\right)+\sqrt{\left(x-x_{j}\right)^{2}+s^{2}}\right) .
\end{aligned}
$$

For $\int d x \int \psi_{i}(x) d x$, which is denoted with $\Phi_{i}(x)$, we have

$$
\begin{aligned}
& \int d x \int \phi_{i}\left(\left|x-x_{i}\right|\right) d x \\
& =\int d x \int \sqrt{s^{2}+\left(x-x_{j}\right)^{2}} d x=\frac{\left(\left(x-x_{j}\right)^{2}+s^{2}\right)^{1.5}}{6} \\
& \quad+\frac{s^{2}}{2}\left(x-x_{j}\right) \ln \left(\left(x-x_{j}\right)+\sqrt{\left(x-x_{j}\right)^{2}+s^{2}}\right) \\
& \quad-\frac{s^{2}}{2} \sqrt{\left(x-x_{j}\right)^{2}+s^{2}} .
\end{aligned}
$$

\section{Numerical Algorithms}

To solve problem (1) and (3), we set

$$
\begin{aligned}
a & =x_{1}<x_{2}<\cdots<x_{m} \\
& =c=x_{m+1}<x_{m+2}<\cdots<x_{m+n} \\
& =d=x_{m+n+1}<x_{m+n+2}<\cdots<x_{m+n+k}=b .
\end{aligned}
$$

Construct $\left(L_{D} f\right)(x)=\sum_{j=1}^{m} f\left(x_{j}\right) \psi_{j}(x)$ on $[a, c]$ to approximate $f(x), x \in[a, c]$; and $\left(L_{D} f\right)(x)=\sum_{j=1}^{k} f\left(x_{m+n+j}\right)$ $\psi_{m+n+j}(x)$ on $[d, b]$ to approximate $f(x), x \in[d, b]$.

On the interval $[c, d]$, let $u^{\prime \prime}(x)=\sum_{j=1}^{n} \alpha_{j} \varphi_{m+j}\left(\left|x-x_{m+j}\right|\right)$, where

$$
\varphi_{m+j}\left(\left|x-x_{m+j}\right|\right)=\frac{s^{2}}{\left(s^{2}+\left(x-x_{m+j}\right)^{2}\right)^{3 / 2}},
$$

and $s$ is a shape parameter and $\alpha_{j}, j=1, \ldots, n$ are coefficients to be identified. We can easily verify that

$$
\begin{gathered}
\int \varphi_{j}\left(\left|x-x_{j}\right|\right) d x=\frac{x-x_{j}}{\sqrt{\left(s^{2}+\left(x-x_{j}\right)^{2}\right)}}+\lambda \\
\int\left(\frac{x-x_{j}}{\sqrt{\left(s^{2}+\left(x-x_{j}\right)^{2}\right)}}+\lambda\right) d x=\phi_{j}\left(\left|x-x_{j}\right|\right)+\lambda x+\mu,
\end{gathered}
$$

where $\lambda, \mu$ are constants. Therefore,

$$
\begin{array}{r}
u(x)=\int d x \int \sum_{j=1}^{n} \alpha_{j} \varphi_{m+j}\left(\left|x-x_{m+j}\right|\right) d x \\
=\sum_{j=1}^{n} \alpha_{j} \phi_{m+j}\left(\left|x-x_{m+j}\right|\right)+\lambda x+\mu, \\
x \in[c, d],
\end{array}
$$

where $\lambda, \mu$ are constants and $\phi_{j}\left(\left|x-x_{j}\right|\right)$ are defined in (15). Therefore, we have the approximation to the solution to problem (1) as follows:

$$
u= \begin{cases}\int d x \sum_{j=1}^{m} f\left(x_{j}\right) \psi_{j}(x) d x & \\ =\sum_{j=1}^{m} f\left(x_{j}\right) \Phi_{j}(x)+c_{1} x+c_{2}, & x \in[a, c], \\ \sum_{j=1}^{n} \alpha_{j} \phi_{m+j}\left(\left|x-x_{m+j}\right|\right) & x \in[c, d], \\ \int d x+\mu, & \\ \int \sum_{j=1}^{k} f\left(x_{m+n+j}\right) \psi_{m+n+j}(x) d x & \\ =\sum_{j=1}^{k} f\left(x_{m+n+j}\right) \Phi_{m+n+j}(x) & x \in[d, b],\end{cases}
$$

where $\Phi_{j}(x)=\int d x \int \psi_{i}(x) d x$ and is computed in (17), and $c_{1}, c_{2}, \lambda, \mu, c_{3}, c_{4}$ are constants. We need to determine the coefficients $c_{1}, c_{2}, \alpha_{1}, \ldots, \alpha_{n}, \lambda, \mu, c_{3}, c_{4}$ to identify the numerical solution (23) to problem (1).

Let

$$
\begin{array}{r}
H_{1}(x)=\sum_{j=1}^{m} f\left(x_{j}\right) \Phi_{j}(x)+c_{1} x+c_{2}, \quad x \in[a, c], \\
H_{2}(x)=\left(\sum_{j=1}^{n} \alpha_{j} \phi_{m+j}\left(\left|x-x_{m+j}\right|\right)+\lambda x+\mu\right), \\
x \in[c, d], \\
H_{3}(x)=\sum_{j=1}^{k} f\left(x_{m+n+j}\right) \Phi_{m+n+j}(x)+c_{3} x+c_{4},
\end{array}
$$


Substitute (24) into problem (1) at points (18) to get the following linear system:

$$
\begin{gathered}
H_{1}(a)=\alpha, \quad H_{1}(c)=H_{2}(c), \\
H_{1}^{\prime}(c)=H_{2}^{\prime}(c), \\
\sum_{j=1}^{n} \alpha_{j} \varphi_{m+j}\left(\left|x_{m+i}-x_{m+j}\right|\right) \\
=g\left(x_{m+i}\right) H_{2}\left(x_{m+i}\right)+f\left(x_{m+i}\right)+r, \\
\quad i=1, \ldots, n, \\
H_{2}(d)=H_{3}(d), \quad H_{2}^{\prime}(d)=H_{3}^{\prime}(d), \\
H_{3}(b)=\beta,
\end{gathered}
$$

where $H_{1}^{\prime}(x), H_{3}^{\prime}(x)$ can be computed in $(16)$ and $H_{2}^{\prime}(x)$ can be computed in (20).

Solving the linear system (24), we get $c_{1}, c_{2}, \alpha_{1}, \ldots, \alpha_{n}, \lambda$, $\mu, c_{3}, c_{4}$ to identify the numerical solution (23) to problem (1).

To solve problem (3), we use

$$
\begin{gathered}
\sum_{j=1}^{m} \alpha_{j} \varphi_{j}\left(\left|x-x_{j}\right|\right), \\
\sum_{j=1}^{n} \alpha_{m+j} \varphi_{m+j}\left(\left|x-x_{m+j}\right|\right), \\
\sum_{j=1}^{k} \alpha_{m+n+j} \varphi_{m+n+j}\left(\left|x-x_{m+n+j}\right|\right)
\end{gathered}
$$

to approximate $u^{\prime \prime \prime}$ on intervals $[a, c],[c, d]$, and $[d, b]$, respectively, where

$$
\varphi_{m+j}\left(\left|x-x_{m+j}\right|\right)=\frac{s^{2}}{\left(s^{2}+\left(x-x_{m+j}\right)^{2}\right)^{3 / 2}}
$$

and $s$ is a shape parameter. To get $u^{\prime \prime}, u^{\prime}$, and $u$, we need only to compute

$$
\begin{gathered}
\int \varphi_{j}\left(\left|x-x_{j}\right|\right) d x=\frac{x-x_{j}}{\left(s^{2}+\left(x-x_{j}\right)^{2}\right)^{1 / 2}}+\lambda, \\
\int d x \int \varphi_{j}\left(\left|x-x_{j}\right|\right) d x=\phi_{j}\left(\left|x-x_{j}\right|\right)+\lambda x+\mu,
\end{gathered}
$$

where $\lambda, \mu$ are constants and $\phi_{j}$ is defined in (13). And we also have

$$
\int\left(\phi_{j}\left(\left|x-x_{j}\right|\right)+\lambda x+\mu\right) d x=\zeta_{j}(x)+\frac{\lambda^{2}}{2} x^{2}+\mu+\nu
$$

where $v$ is a constant and

$$
\begin{aligned}
\zeta_{j}(x)= & \frac{\left(x-x_{j}\right) \sqrt{\left(x-x_{j}\right)^{2}+s^{2}}}{2} \\
& +\frac{s^{2}}{2} \ln \left(\left(x-x_{j}\right)+\sqrt{\left(x-x_{j}\right)^{2}+s^{2}}\right) .
\end{aligned}
$$

Therefore, we need to identify the following numerical solution to (3):

$$
u=\left\{\begin{array}{l}
\sum_{j=1}^{m} \alpha_{j} \zeta_{j}(x)+c_{1} x^{2}+c_{2} x+c_{3}, \\
\sum_{j=1}^{n} \alpha_{m+j} \zeta_{m+j}(x)+\lambda x^{2}+\mu x+\nu, \\
\sum_{j=1}^{k} \alpha_{m+n+j} \zeta_{m+n+j}(x)+c_{4} x^{2}+c_{5} x+c_{6} .
\end{array}\right.
$$

To determine the coefficients $c_{1}, c_{2}, c_{3}, \alpha_{1}, \ldots, \alpha_{m+n+k}, \lambda, \mu, \nu$, $c_{3}, c_{4}, c_{5}$, denote

$$
\begin{gathered}
H_{1}(x)=\sum_{j=1}^{m} \alpha_{j} \zeta_{j}(x)+c_{1} x^{2}+c_{2} x+c_{3}, \\
H_{2}(x)=\sum_{j=1}^{n} \alpha_{m+j} \zeta_{m+j}(x)+\lambda x^{2}+\mu x+\nu, \\
H_{3}(x)=\sum_{j=1}^{k} \alpha_{m+n+j} \zeta_{m+n+j}(x)+c_{4} x^{2}+c_{5} x+c_{6},
\end{gathered}
$$

and $H_{1}^{\prime}(x), H_{2}^{\prime}(x), H_{3}^{\prime}(x)$ can be computed in (29) and $H_{1}^{\prime \prime}(x)$, $H_{2}^{\prime \prime}(x), H_{3}^{\prime \prime}(x)$ can be computed in (28). Substitute (25) into problem (3) at points (18) to get the following linear system:

$$
\begin{gathered}
H_{1}(a)=\alpha_{1}, \quad H_{1}^{\prime}(a)=\alpha_{2}, \\
H_{1}(c)=H_{2}(c), \quad H_{1}^{\prime}(c)=H_{2}^{\prime}(c), \\
H_{1}^{\prime \prime}(c)=H_{2}^{\prime \prime}(c), \\
\sum_{j=1}^{n} \alpha_{m+j} \varphi_{m+j}\left(\left|x_{m+i}-x_{m+j}\right|\right) \\
=g\left(x_{i}\right) H_{2}\left(x_{m+i}\right)+f\left(x_{m+i}\right)+r, \\
H_{2}(d)=H_{3}(d), \quad H_{2}^{\prime}(d)=H_{3}^{\prime}(d), \\
H_{2}^{\prime \prime}(d)=H_{3}^{\prime \prime}(d), \quad H_{3}^{\prime}(b)=\beta .
\end{gathered}
$$

Then, we can solve (34) to identify the numerical solution (32) to problem (3).

\section{Numerical Experiment}

We do numerical experiments to test the efficiency of our numerical methods. 
TABLE 1: Comparison of exact and numerical solutions for Example 1.

\begin{tabular}{lccc}
\hline$x$ & $s=1.0, m=n=6$ & Exact solution & $s=1.2, m=n=9$ \\
RBF method & 0 \\
\hline$x_{1}$ & 0 & 0 & 0.13596345616525 \\
$x_{3}$ & 0.13633712736 & 0.13598397716525 & 0.33994454291217 \\
$x_{6}$ & 0.34084281842 & 0.33995994291313 & 0.44536178581320 \\
$x_{7}$ & 0.44620383234 & 0.44535138581350 & 0.49553678904558 \\
$x_{8}$ & 0.49629810661 & 0.49554948904960 & 0.49553578798663 \\
$x_{9}$ & 0.49629810661 & 0.49554948904960 & 0.44536480583846 \\
$x_{10}$ & 0.44620383234 & 0.44535138581350 & 0.33996457654310 \\
$x_{11}$ & 0.34084281842 & 0.33995994291313 & 0.13597686714765 \\
$x_{14}$ & 0.13633712736 & 0.13598397716525 & 0 \\
$x_{16}$ & 0 & 0 & $1.54000009599 e-005$ \\
$L_{\infty}$ error & $8.82875509702 e-004$ & & $3.69805440717 e-006$ \\
$L_{2}$ error & $2.09345530306 e-004$ & & \\
\hline
\end{tabular}

TABLE 2: Comparison of exact and numerical solutions for Example 2.

\begin{tabular}{lccc}
\hline$x$ & $\begin{array}{c}s=1.0, m=n=6 \\
\text { RBF method }\end{array}$ & Exact solution & $\begin{array}{c}s=1.2, m=n=9 \\
\text { RBF method }\end{array}$ \\
\hline 0.0 & 0 & 0 & 0 \\
0.1 & 0.00078568857778 & 0.00072603711810 & 0.00072504958690 \\
0.2 & 0.00294888593935 & 0.00290414847242 & 0.00290536784209 \\
0.3 & 0.00653975857589 & 0.00651360507585 & 0.00651038335789 \\
0.4 & 0.01101039579013 & 0.01105748825987 & 0.01105193964378 \\
0.5 & 0.01550387591395 & 0.01556533144116 & 0.01556193848997 \\
0.6 & 0.01909384653746 & 0.01905044604835 & 0.01905677849423 \\
0.7 & 0.02053875436899 & 0.02053013998469 & 0.02053284784996 \\
0.8 & 0.01951387899385 & 0.01955230368928 & 0.01955135789349 \\
0.9 & 0.01862948575889 & 0.01867323762406 & 0.01867204968224 \\
1.0 & 0.01832948588680 & 0.01838021560231 & 0.01838334510084 \\
$L_{\infty}$ error & $6.145552721 e-005$ & & $6.3324458800 e-006$ \\
$L_{2}$ error & $1.290788173 e-005$ & & $9.7350311797 e-007$ \\
\hline
\end{tabular}

Example 1. Consider the following second-order boundary obstacle problem as given in [22]:

$$
u^{\prime \prime}= \begin{cases}0, & 0 \leq x \leq \frac{\pi}{4}, \\ u-1, & \frac{\pi}{4} \leq x \leq \frac{3}{4} \pi, \\ 0, & \frac{3}{4} \pi \leq x \leq \pi,\end{cases}
$$

with boundary condition $u(0)=0, u(\pi)=0$. The exact solution of this problem is given as

$$
u= \begin{cases}\frac{4}{r_{1}} x, & 0 \leq x \leq \frac{\pi}{4}, \\ 1-\frac{4}{r_{2}} \cosh \left(\frac{\pi}{4}-x\right), & \frac{\pi}{4} \leq x \leq \frac{3}{4} \pi, \\ \frac{4}{r_{1}}(\pi-x), & \frac{3}{4} \pi \leq x \leq \pi,\end{cases}
$$

where $r_{1}=\pi+4 \operatorname{coth}(\pi / 4), r_{2}=\pi \sinh (\pi / 4)+4 \cosh (\pi / 4)$.
The results are reported in Table 1 . The errors between numerical and exact solution in terms of $L_{\infty}$ and $L_{2}$ are listed in Table 1 . The accuracy of the proposed method is measured using the $L_{2}$ and $L_{\infty}$ error norms defined as

$$
\begin{gathered}
L_{2}=\frac{1}{n+1} \sqrt{\sum_{i=0}^{n}\left|u_{i}^{\text {exact }}-u_{i}^{\text {num }}\right|^{2}}, \\
L_{\infty}=\max _{0 \leq i \leq n}\left|u_{i}^{\text {exact }}-u_{i}^{\text {num }}\right| .
\end{gathered}
$$

In Table 1, we set

$$
\begin{gathered}
x_{i}=(i-1) h_{1}, \quad i=1,2, \ldots, 6, h_{1}=\frac{\pi}{20}, \\
x_{i}=\frac{\pi}{4}+(i-6) h_{2}, \quad i=6,7, \ldots, 11, h_{2}=\frac{\pi}{10}, \\
x_{i}=\frac{3}{4} \pi+(i-11) h_{1}, \quad i=12,13, \ldots, 17, h_{1}=\frac{\pi}{20} .
\end{gathered}
$$


Example 2. Consider the following third-order boundary obstacle problem as given in [17]:

$$
u^{\prime \prime \prime}(x)= \begin{cases}0, & 0 \leq x \leq \frac{1}{4} \\ u(x)-1, & \frac{1}{4} \leq x \leq \frac{3}{4} \\ 0, & \frac{3}{4} \leq x \leq 1\end{cases}
$$

with boundary conditions $u(0)=0, u^{\prime}(0)=0, u^{\prime}(1)=0$.

The exact solution of this problem is

$$
u(x)=\left\{\begin{array}{cc}
\frac{1}{2} a_{1} x^{2}, & 0 \leq x \leq \frac{1}{4}, \\
1+a_{2} e^{x} & +e^{-x / 2}\left(a_{3} \cos \frac{\sqrt{3}}{2} x\right. \\
\left.+a_{4} \sin \frac{\sqrt{3}}{2} x\right), & \frac{1}{4} \leq x \leq \frac{3}{4}, \\
a_{5} x\left(\frac{1}{2} x-1\right)+a_{6}, & \frac{3}{4} \leq x \leq 1,
\end{array}\right.
$$

where

$$
\begin{aligned}
& a_{1}=0.14520742362098 \\
& a_{2}=-0.21130240827197 \\
& a_{3}=-0.78610085318732 \\
& a_{4}=-0.24585768969643 \\
& a_{5}=0.05860440434801 \\
& a_{6}=0.04768241777632
\end{aligned}
$$

The results are reported in Table 2 . The errors between numerical and exact solution in terms of $L_{\infty}$ and $L_{2}$ are listed in Table 2.

From the results of Tables 1 and 2, we can see that the shape parameter $s$ plays no significant role in the computation.

\section{Conclusions}

In this paper, based on the multiquadric RBF and inverse multiquadric RBF, numerical schemes are presented for solving system of second-order and third-order boundary value problems associated with obstacle. The schemes are shown to be highly accurate and easy to implement. We point out three notes here. First, to solve second-order problem, we use $L_{D}$ operator to approximate the derivatives on the interval $[a, c]$ and $[d, b]$ because by using $L_{D}$ operator, we can on one hand avoid solving linear systems and on the other hand achieve high accuracy. Second, to solve third-order problem, we do not employ $L_{D}$ operators to approximate the derivatives on the interval $[a, c]$ and $[d, b]$ because it is no easy to integrate $L_{D} f$ for three times and no explicit integral expressions can be found. Third, why we choose MQs and IMQs rather than other RBF? That is because
MQs and IMQs can achieve high accuracy and IMQs are the second derivatives of MQs and this property bring much convenience to our calculation. Further study can be carried out for the applications of RBF in the numerical solution of higher-order boundary value obstacle problems.

\section{References}

[1] C. Baiocchi and A. Capelo, Variational and Quasivariational Inequalities, John Wiley \& Sons, New York, NY, USA, 1984.

[2] F. Giannessi and A. Magueri, Variational Inequalities and Network Equilibrium Problems, Plenum Press, New York, NY, USA, 1995.

[3] M. A. Noor, K. I. Noor, and T. M. Rassias, "Some aspects of variational inequalities," Journal of Computational and Applied Mathematics, vol. 47, no. 3, pp. 285-312, 1993.

[4] E. A. Al-Said, "Smooth spline solutions for a system of second order boundary value problems," Journal of Natural Geometry, vol. 16, no. 1-2, pp. 19-28, 1999.

[5] E. A. Al-Said, "Spline solutions for system of second-order boundary-value problems," International Journal of Computer Mathematics, vol. 62, no. 1-2, pp. 143-154, 1996.

[6] E. A. Al-Said, "Spline methods for solving system of secondorder boundary-value problems," International Journal of Computer Mathematics, vol. 70, no. 4, pp. 717-727, 1999.

[7] E. A. Al-Said, "The use of cubic splines in the numerical solution of a system of second-order boundary value problems," Computers \& Mathematics with Applications, vol. 42, no. 6-7, pp. 861-869, 2001.

[8] M. Aslam Noor, "Some developments in general variational inequalities," Applied Mathematics and Computation, vol. 152, no. 1, pp. 199-277, 2004.

[9] M. A. Noor, Principles of Variational Inequalities, Lap-Lambert Academic Publishing AG, Saarbrücken, Germany, 2009.

[10] M. A. Noor, K. I. Noor, A. Waheed, and E.-A. Eisa-Al-Said, "Modified variation of parameters method for solving system of second-order nonlinear boundary value problem," International Journal of Physical Sciences, vol. 5, no. 16, pp. 2426-2431, 2010.

[11] M. A. Noor and S. I. A. Tirmizi, "Numerical methods for unilateral problems," Journal of Computational and Applied Mathematics, vol. 16, no. 3, pp. 387-395, 1986.

[12] Siraj-ul-Islam, M. A. Khan, I. A. Tirmizi, and E. H. Twizell, "Non polynomial spline approach to the solution of a system of third-order boundary-value problems," Applied Mathematics and Computation, vol. 168, no. 1, pp. 152-163, 2005.

[13] M. A. Noor and A. K. Khalifa, "Cubic splines collocation methods for unilateral problems," International Journal of Engineering Science, vol. 25, no. 11-12, pp. 1525-1530, 1987.

[14] E. A. Al-Said, M. A. Noor, and A. A. Al-Shejari, "Numerical solutions for system of second order boundary value problems," The Korean Journal of Computational \& Applied Mathematics, vol. 5, no. 3, pp. 659-667, 1998.

[15] A. K. Khalifa and M. A. Noor, "Quintic splines solutions of a class of contact problems," Mathematical and Computer Modelling, vol. 13, no. 2, pp. 51-58, 1990.

[16] M. A. Noor and A. K. Khalifa, "A numerical approach for oddorder obstacle problems," International Journal of Computer Mathematics, vol. 54, no. 1-2, pp. 109-116, 1994. 
[17] F. Gao and C.-M. Chi, "Solving third-order obstacle problems with quartic B-splines," Applied Mathematics and Computation, vol. 180, no. 1, pp. 270-274, 2006.

[18] E. Larsson and B. Fornberg, "A numerical study of some radial basis function based solution methods for elliptic PDEs," Computers \& Mathematics with Applications. An International Journal, vol. 46, no. 5-6, pp. 891-902, 2003.

[19] M. D. Buhmann, S. Dinew, and E. Larsson, "A note on radial basis function interpolant limits," IMA Journal of Numerical Analysis, vol. 30, no. 2, pp. 543-554, 2010.

[20] D. G. Krige, A statistical approach to some mine valuation and allied problems on the Witwatersrand, [M.Sc. thesis], University of Witwatersrand, Witwatersrand, South Africa, 1951.

[21] C. A. Micchelli, "Interpolation of scattered data: distance matrices and conditionally positive definite functions," Constructive Approximation, vol. 2, no. 1, pp. 11-22, 1986.

[22] P. Villaggio, "The Ritz method in solving unilateral problems in elasticity," Meccanica, vol. 16, no. 3, pp. 123-127, 1981. 


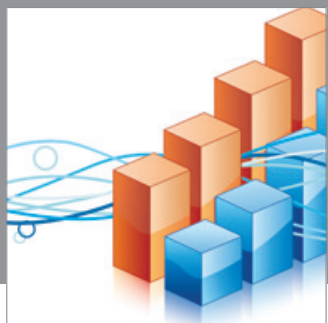

Advances in

Operations Research

mansans

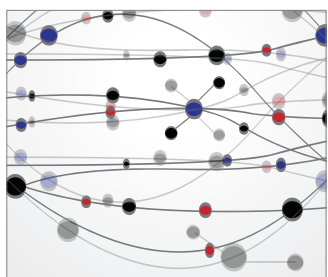

The Scientific World Journal
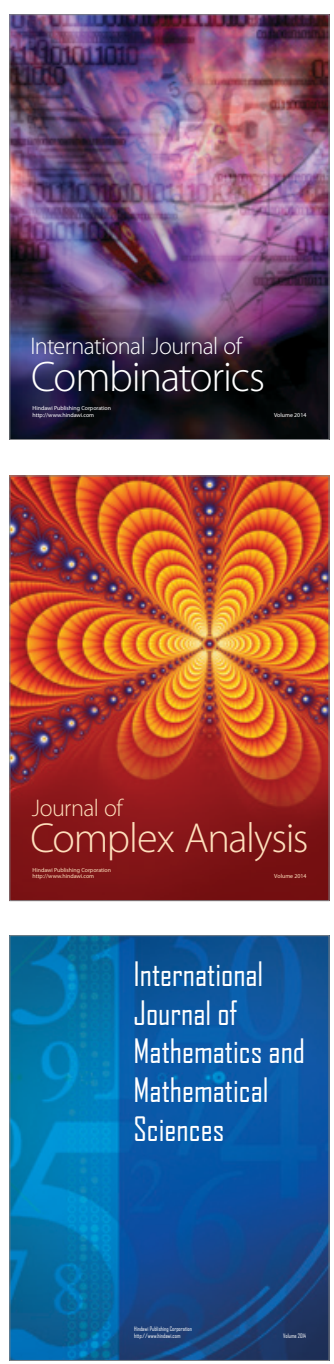
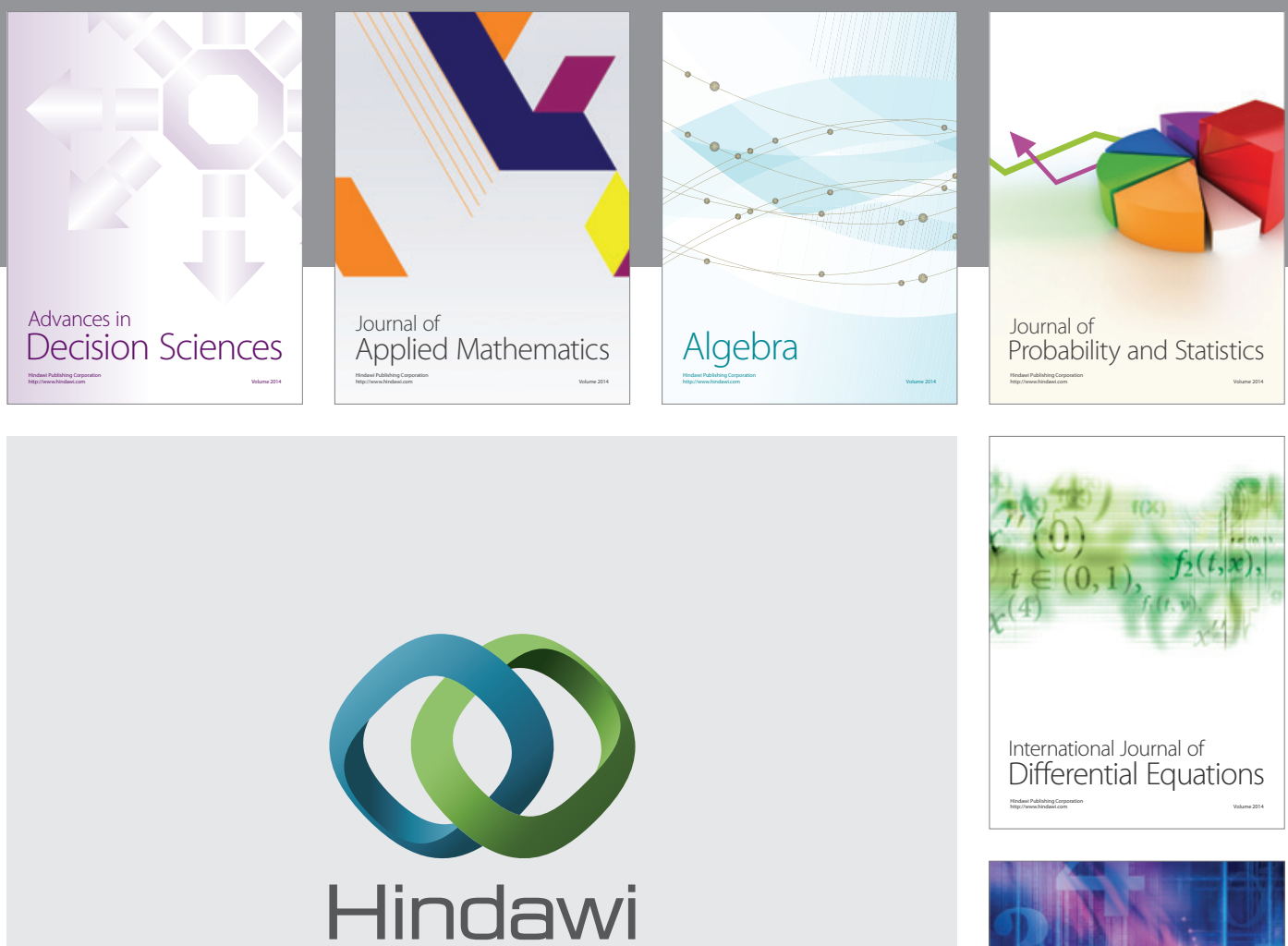

Submit your manuscripts at http://www.hindawi.com
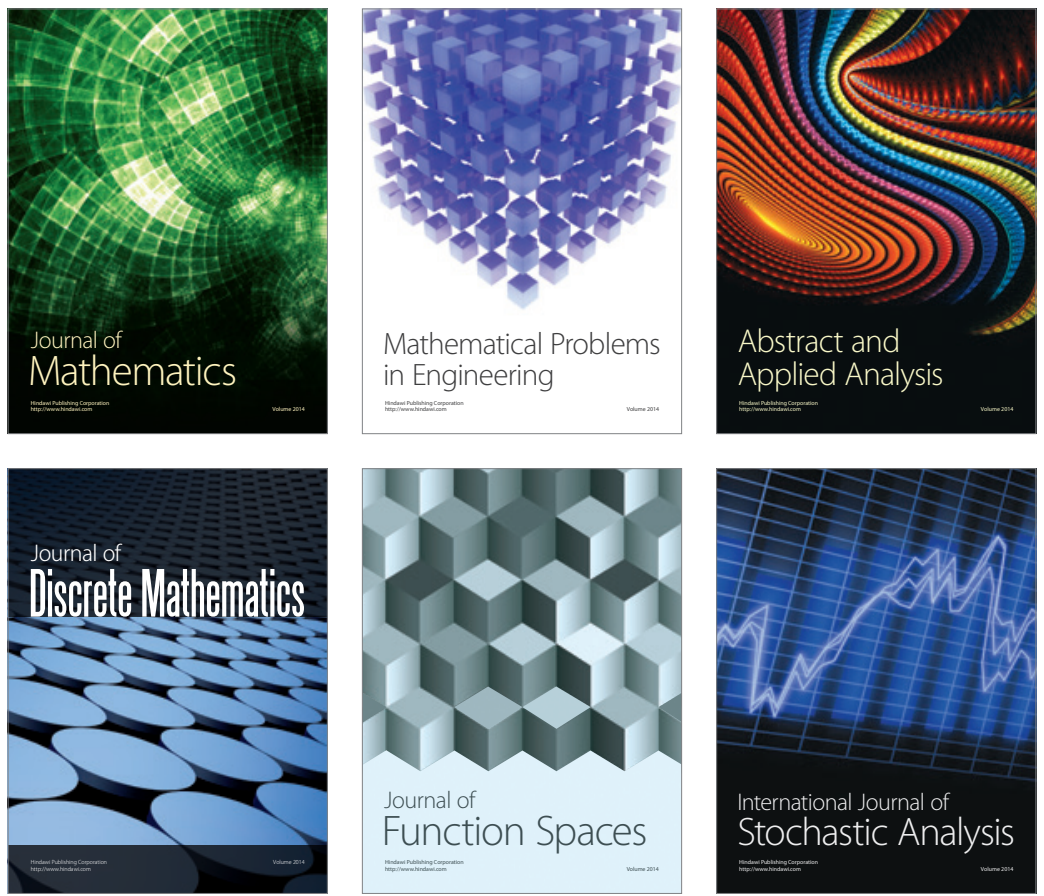

Journal of

Function Spaces

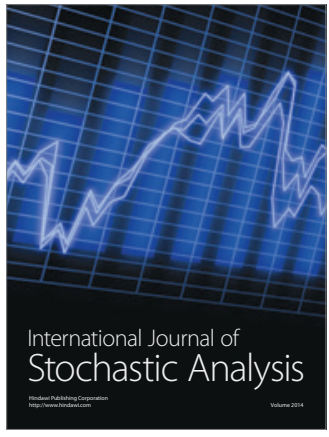

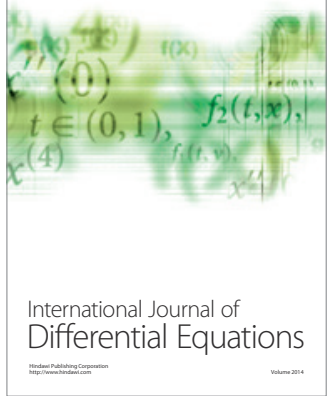
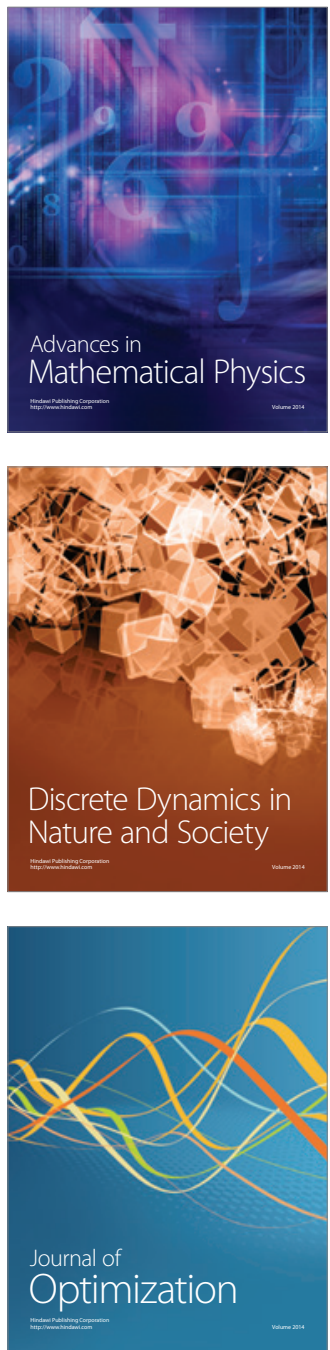